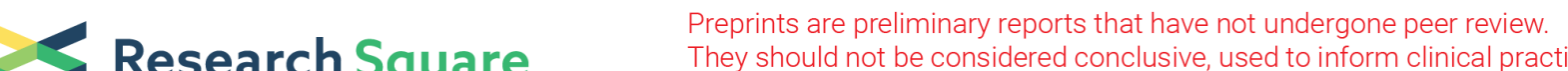 Research Square They should not be considered conclusive, used to inform clinical practice, or referenced by the media as validated information.
}

\section{Microstructure Evolution of (MgCoNiZnCu)O High Entropy Oxides Using Ceramic Injection Molding}

\section{Yipeng ZHAO}

Dalian University of Technology

Guoqing CHEN ( $\nabla$ gqchen@dlut.edu.cn )

Dalian University of Technology https://orcid.org/0000-0002-6695-2587

Hongwei LI

Dalian University of Technology

Xuesong FU

Dalian University of Technology

Wenlong ZHOU

Dalian University of Technology

\section{Research Article}

Keywords: high entropy oxides, rock-salt structure, near net shaping process, cost-effective

Posted Date: September 15th, 2021

DOI: https://doi.org/10.21203/rs.3.rs-855939/v1

License: (c) (i) This work is licensed under a Creative Commons Attribution 4.0 International License. Read Full License 


\section{Abstract}

Near net shaping ceramic injection molding process of $(\mathrm{MgCoNiZnCu}) \mathrm{O}$ high entropy oxides were conducted using commercial precursor oxide powders. Through ball milling, internal mixing, injection molding, solvent and thermal debinding as well as final sintering process, the ceramic products would be obtained with little machining. Compacts prepared are single rock-salt phase based on XRD and EDS Mapping results. Meanwhile, with the increasing of sintering temperature from $900{ }^{\circ} \mathrm{C}$ to $1050{ }^{\circ} \mathrm{C}$, particle diffusion rate and densification of samples becomes faster, which finally results relative density and fractured strength of sintered compacts reaching the highest $(90.47 \%$ and $77.98 \mathrm{MPa}$, respectively) in current work. The successfully synthesis of ( $\mathrm{MgCoNiZnCu}) 0$ through ceramic injection molding illustrates this near net shaping process could be a promising route for preparation of high entropy oxides.

\section{Highlights}

A cost effective near net shaping method for (MgCoNiZnCu)O high entropy oxides was proposed.

(MgCoNiZnCu)O samples synthesized are single rock-salt phase with homogeneous microstructure.

The relative density of ceramic injection molding compacts reaches $90.47 \%$.

\section{Introduction}

The 'high entropy' material was first introduced by Yeh et al for described high entropy alloys (HEA) which is composed of five or more metal elements in equimolar or near equimolar ratios[1]. An $\mathrm{N}$-species material system with composition $\left(x_{i}\right)$ has ideal configurational entropy $S[2]$ :

$$
S=-\mathrm{R} \sum_{i=1}^{N} x_{i} \log \left(x_{i}\right)
$$

Maximum $S_{\max }$ is acquired at stoichiometric amounts, as $x_{i}=1 / \mathrm{N}$ for each $i$, hence:

$S_{\max }=\mathrm{R} \log (M)(2)$

In regard to a high entropy system, it is well known that configurational entropy is always not less than $1.61 \mathrm{R}[3]$.

Rost et al. extended the HEA concept to the field of ceramics and successfully synthesized a rock-salt structure (MgCoNiZnCu)O high entropy oxide (HEO) in 2015[2]. The HEO rock-salt phase has two different kinds of sublattices: the oxygen particles occupy the sites of face centered cubic lattice while the five kinds of cations locate at the octahedral gaps of the oxygen atoms randomly and uniformly[4,5]. Since then, $\mathrm{HEO}$ especially (MgCoNiZnCu)O has been thoroughly studied because of colossal dielectric constant[6], exceptional lithium-ion conductivity[7], excellent magnetic properties[8] and catalytic 
performance[9]. The incorporation of different metal cations in a single lattice structure, their possible synergistic effects, and the larger configurational entropy contribute to such multifunctional properties for this type of materials. Besides, much attention has been focused on synthesis of HEO and its derivatives[10], with methods including solid-state reaction[6], nebulized spray pyrolysis[4], flame spray pyrolysis[4], co-precipitation[11] and solution combustion method[12].

Among the large quantity of existing synthesis process for advanced ceramics, it is worth noting that ceramic injection molding (CIM) could overcomes limitations of conventional procedures due to a series of outstanding advantages. As a cost-effective near net shaping process, CIM attracts wide intention in recent years which could manufacture small, complex shaped, high dimensional precision ceramic compacts in enormous quantities[13-15]. This technology is usually comprised of four basic stages: compounding of feedstock, injection molding, debinding and sintering[16,17]. Feedstock of CIM usually consists of organic binders and ceramic powders. The binder system is a complex mixture composed of several components which is strictly selected to facilitated well injection properties and can be easily removed from green parts[18]. Binders used in CIM mainly consists of three functional components including: lubricants providing rheological properties for feedstock, macromolecular binders maintaining the shape integrity of green parts and debound compacts, as well as surfactants improving compatibility between ceramic powders and other organic binders[19]. CIM technology was used to fabricate a series of ceramic materials as follows: $\mathrm{Al}_{2} \mathrm{O}_{3}$ [20,21], $\mathrm{ZrO}_{2}$ [19,22], $\mathrm{CaO}$ [18], $\mathrm{SiO}_{2}$ [23], $\mathrm{MgAl}_{2} \mathrm{O}_{4}$ [24,25], $\mathrm{SiC}[26]$, $\mathrm{B}_{4} \mathrm{C}$ [27], $\mathrm{AIN}$ [13], $\mathrm{Si}_{3} \mathrm{~N}_{4}$ [28] and $\mathrm{ZrB}_{2}$ [29] et al. Through this near net shaping CIM process, a large quantity of ceramic parts have been synthesized in recent years. Qin et al. [13] reported the CIM of complex shaped AIN ceramic with high thermal conductivity and successfully synthesized AIN heat sinks. Gromada et al. [30] have obtained materials allowed for the formation of ceramic cores with complicated shapes and high dimensional accuracy. While Hu et al. [20] fabricated a series of translucent alumina ceramics as prefabricated dental post, orthodontic dental ceramic bracket and relatively large size ceramic bowls using surface modificated alumina powders.

In accordance with the best of our authors' knowledge, despite the previously described superiority of CIM technology, it has been never used to synthesize multi-components high entropy materials especially ( $\mathrm{MgCoNiZnCu}$ ) O HEO. In light of this aspect, a single rock-salt structure ( $\mathrm{MgCoNiZnCu}$ ) O was successfully synthesized for the first time using near net shaping CIM technology in present study. Besides, the Microstructure evolution of (MgCoNiZnCu)O was systematically discussed to provide process guidance for the future work about the synthesize of HEO through CIM.

\section{Experimental}

\subsection{Preparation of (MgCoNiZnCu)O}

(MgCoNiZnCu)O HEO was prepared from MgO (99.9\% purity, $2.5 \mu \mathrm{m}), \mathrm{CoO}(99.9 \%$ purity, $1 \mu \mathrm{m}), \mathrm{NiO}$ (99.9\% purity, $1 \mu \mathrm{m}), \mathrm{ZnO}(99.9 \%$ purity, $1 \mu \mathrm{m})$ and CuO (99.9\% purity, $1 \mu \mathrm{m})$ acquired from Shanghai STNano Science \& Technology Co., Ltd. The process scheme for synthesis of (MgCoNiZnCu)O by CIM is 
present in Fig. 1. At the first mixing stage shown in Fig. 1, ceramic powders with stoichiometric amounts and few stearic acids (SA) in ethanol were mechanically mixed by planetary ball milling (QM-3SP4) using alumina balls and vials at $300 \mathrm{rpm}$ for 6 hours. Afterward dried powders (morphology and XRD pattern were shown in Fig. S1 and Fig. S2, respectively) were mixed with organic binders (polyethylene (PE), polypropylene (PP), paraffin wax (PW) and dibutyl phthalate (DBP)) in 50 vol\% powder loading using an internal mixer machine (KY-3220A-1L) to prepare feedstock on $170{ }^{\circ} \mathrm{C}$ for $45 \mathrm{~min}$ at $40 \mathrm{rpm}$. Secondly, at injection molding stage, green parts $(5 \times 6 \times 42 \mathrm{~mm})$ were prepared using an injection molding machine (V35-CIM) with injection temperature and pressure were $180^{\circ} \mathrm{C}$ and $110 \mathrm{MPa}$, respectively. Then at debinding stage, a two stage debinding process (solvent and thermal) was used to remove binder system in green parts. Hence green parts were immersed in kerosene (nontoxic, recycled and cheap) and heated to $55^{\circ} \mathrm{C}$ in a water bath for 8 hours to partially remove soluble PW. Compacts after solvent debinding were heated to $500{ }^{\circ} \mathrm{C}$ for 2 hours in a debinding furnace (QH-12-11) with heating and cooling rate of 1.5 ${ }^{\circ} \mathrm{C} /$ min (according to TG results of Fig. 2a) to remove remaining binders. Finally at the sintering stage, compacts were transferred to a tube furnace (GSL-1500X-OTF) for sintering at 900 (about $20^{\circ} \mathrm{C}$ higher than weight loss temperature of Fig. 2c), $1000,1050{ }^{\circ} \mathrm{C}$ (a little lower than weight loss temperature of Fig. $2 \mathrm{e}$ ) with holding time of $6,10,18$ hours, respectively.

\subsection{Characterization and measurements}

Thermogravimetric (TG) and differential scanning calorimetry (DSC) experiments about mixed powder, green part and solvent debound part were carried out using a thermogravimetric analyzer (TGA/SDTA851e, Switzerland). While crystal structure of sintered samples was characterized using X-ray diffractometer (XRD-6000, Japan) equipped with Cu Ka source at $4 \%$ min scanning speed and $20-80^{\circ}$ scanning range. Analysis of polished and fractured surface was conducted by scanning electron microscopy (SEM, SUPRA 55, Germany), while selected area was characterized by energy dispersive spectroscopy (EDS, Germany) to confirm elements distribution uniformity. Grain size of compacts was estimated from micrograph of fractured surface using Nano Measurer software. According to Archimedes' principle, bulk density of sintered pellets was measured in deionized water, while compression strength was evaluated using a universal testing machine (DNS 300, China) with a crosshead speed of $0.2 \mathrm{~mm} / \mathrm{min}$.

\section{Results And Discussion}

\subsection{Organic binder}

Being one of the important constituents in process of CIM, organic binders always consist of several binders: low molecular weight polymers as lubricants, macromolecular binders as backbone binders as well as a small amount of surfactant[16,22]. As a surfactant, SA used in this work has both functional group anchoring to the powder surface and the carbon chain dissolving in the binders[21], hence could improve the compatibility between binders and oxide powders. During the planetary ball milling progress, the SA has been coated on the surface of oxide powders[22], and furtherly make the organic binders tightly coated on the surface of the modificated oxide powders after internal mixing process. While the 
lubricants (PW and DBP in this work) could improve fluidity of feedstock and enhance the wettability between organic binders and mixed powders. The lubricants also endow feedstock enough rheological properties during the injection stage for better molding abilities of complex shaped green parts. Besides, the backbone binders (PE and PP) give mechanical strength to green compacts as well as pellets after solvent debinding to maintain their shapes. Though the crucial function of organic binders at the injection and debinding stage, it is harmful at the sintering stage. Hence the combination of solvent and thermal debinding process will remove the binder system from the green parts rapidly and avoid defects. Besides, thermal properties were analyzed and evaluated through TG and DSC experiments for debinding process to accelerate the removal rate and eliminate crack defects[31].

TG results present three stages of weight loss from 150 to $1200^{\circ} \mathrm{C}$, data of DSC also show three stages in similar temperature range. Firstly, greatest weight loss occurs between 210 to $480{ }^{\circ} \mathrm{C}$ (present in Fig. 2a) due to the removal of organic binders. Detailed analysis can be gained from the DSC results of Fig. $2 \mathrm{~b}$ as follows: the first peak from 210 to $240{ }^{\circ} \mathrm{C}$ shows decomposition of low molecular weight DBP and PW binders (mainly the lubricants), the removal temperature of SA reaches $280{ }^{\circ} \mathrm{C}$ due to great affinity between the surfactant SA and mixed powders, and peaks from 300 to $480^{\circ} \mathrm{C}$ display the elimination of backbone binders PE and PP whose melting point is higher[16]. Therefore in the process of thermal debinding, the slow heating rate of $1.5^{\circ} \mathrm{C} / \mathrm{min}$ was adopted in order to maintain the complete shape and defect free of the CIM compacts. It should be stated that the weight loss of mixed powder is owing to the SA introduced from planetary ball milling for the effect of surface modification. The formation of single rock-salt solid solution high entropy structure locates at $690-880^{\circ} \mathrm{C}$ explains weight loss of mixed powder, green part as well as solvent debound part shown in Fig. 2c. And from this it can be concluded that binder system was completely removed from the compacts at the first weight loss stage. Considering the former discussion, the lowest sintering temperature (locates at $900{ }^{\circ} \mathrm{C}$ ) of this work is a little higher than the former temperature range $\left(690-880^{\circ} \mathrm{C}\right)$. The weight loss from 1100 to $1200{ }^{\circ} \mathrm{C}$ in Fig. $2 \mathrm{~d}$ features the elimination of $\mathrm{CuO}$, so the highest sintering temperature (set as $1050{ }^{\circ} \mathrm{C}$ ) is lower than 1100 ${ }^{\circ} \mathrm{C}$ in this work.

\subsection{Analysis of green and debound parts}

The quality of green and debound parts will influence the quality of the final sintered CIM parts, hence the morphology of green and solvent debound parts was characterized and systematically analyzed for the purpose of defect free final parts. Figure 3(a-c) shows the surface and fractured micrograph of CIM green parts, respectively. It suggests that green parts are tightly packed by uniformly distributed ceramic powers fully surrounded by organic binders. Figure $3 \mathrm{c}$ presents a large quantity of fibrous binders of the fractured morphology, which is mainly caused by the elongation of organic binders in the process of fracture. Figure 3(d-f) responds to images of solvent debound samples. After partly removal of soluble PW, some pore channels were observed in compacts when compare Fig. 3d with Fig. 3a, and it could serve as escape paths for decomposed gas during subsequent thermal debinding for backbone binders[32]. After solvent debinding, there were about $10 \%$ solvent removable binders remaining in compacts except backbone binders, then subsequent thermal debinding would remove the remaining organic binders. 
Finally, a sintering operation according to the parameters present in the former experimental process of this article is carried out on the debound parts to achieve the required physical and mechanical properties[33].

\subsection{Morphologies and Microstructure evolution}

After the preparation of sintered CIM compacts, the XRD and SEM tests are conducted to confirm the formation of HEO. XRD spectra of final compacts present in Fig. 4a illustrate that (MgCoNiZnCu)O samples sintered at 900,1000 and $1050{ }^{\circ} \mathrm{C}$ using CIM process are single phase, with all Bragg peaks can be indexed in rock-salt Fm-3m (225) space group which is consistent with the research results of Christina M. Rost[2]. Besides, when sintered at $1050{ }^{\circ} \mathrm{C}$, SEM images of polished morphology and corresponding EDS mapping of $\mathrm{Mg}, \mathrm{Co}, \mathrm{Ni}, \mathrm{Zn}, \mathrm{Cu}$ and $\mathrm{O}$ elements shown in Fig. 4b illustrates that the distribution of metallic and nonmetallic elements is random and uniform at this micron scale. And there exists none segregation and gathering of all constituent elements confirming the chemical and microstructural homogeneity. Given the above discussion, the XRD and EDS mapping results demonstrate that single rock-salt phase (MgCoNiZnCu)O HEO has been synthesized using near net shaping CIM process.

Sintering temperature also influences densification behavior of samples except holding time (discussed about Fig. S3). SEM micrographs of fractured samples experienced different temperature are presented in Fig. 5(a-f). At $900{ }^{\circ} \mathrm{C}$, fractured surface indicates the package of grains is loose and the connection between grains is pretty weak as shown in Fig. $5(\mathrm{a}, \mathrm{b})$. While compressive fractured strength of this compact is only 22.29 MPa listed in Table 1 though the mean grain size is only $2.60 \mu \mathrm{m}$ in Fig. $5 \mathrm{~g}$. As a matter of fact, the removal of organic binders leaves a large quantity of micro holes in compacts after the two stage debinding process, the powder loading is 50 vol\% for enough formability of ceramic injection molding process, hence there are probably 50 vol\% of holes in thermal debinding samples. Despite the formation of single rock-salt phase has been achieved according to the XRD result of Fig. 4a prepared at $900{ }^{\circ} \mathrm{C}$, the densification of compacts still needs higher sintering temperature. When the temperature of $1000{ }^{\circ} \mathrm{C}$ was reached (Fig. 5(c, d)), connections between particles become stronger but there are still some holes distributing on fractured surface, while the fractured strength and mean grain size are 58.13 $\mathrm{MPa}$ and $3.78 \mu \mathrm{m}$, respectively. At the highest sintering temperature of this work $\left(1050{ }^{\circ} \mathrm{C}\right), \mathrm{Fig} .5(\mathrm{e}, \mathrm{f})$ is characterized by a dense morphology and tightly bonded grains, with relative density above $90.47 \%$. Hence fractured strength reaches the highest value (77.98 MPa) though grains have some roughening and the mean grain size reaches $5.56 \mu \mathrm{m}$. With increasing of sintering temperature, diffusion rate of particles and densification of pellets become faster, finally results the relative density as well as fractured strength reaching the highest at present study. 
Table 1

Physical properties of sintered compacts

\begin{tabular}{|llll|}
\hline Sintering Temperature $\left({ }^{\circ} \mathrm{C}\right)$ & 900 & 1000 & 1050 \\
\hline Density $\left(\mathrm{g} . \mathrm{cm}^{-3}\right)$ & 5.0499 & 5.2889 & 5.5821 \\
\hline Relative density (\%) & 82.72 & 85.01 & 90.47 \\
\hline Fracture strength (MPa) & 22.29 & 58.13 & 77.98 \\
\hline
\end{tabular}

\section{Conclusions}

Results indicate that the TG and DSC analysis illustrate the removal mechanism of organic binders during thermal debinding experiment and the defect free brown parts were gained with a shorter process time. Micrograph of green and solvent debound parts was characterized for the purpose of obtaining defect free final parts. Then a single rock-salt phase (MgCoNiZnCu)O was successfully synthesized through CIM process. The highest value of fractured strength $(77.98 \mathrm{MPa})$ was achieved for ( $\mathrm{MgCoNiZnCu}$ ) O sintered at $1050{ }^{\circ} \mathrm{C}$, with the relative density of final HEO compact reaches $90.47 \%$. Overall, this research clearly demonstrates that CIM is a pretty promising cost effective near net shaping technique to synthesis HEO.

\section{Declarations}

\section{CRediT authorship contribution statement}

Yipeng ZHAO: Investigation, Validation, Methodology, Writing, Visualization, Review \& Editing.

Guoqing CHEN: Conceptualization, Resources, Validation, Review \& Editing, Supervision, Visualization, Project administration, Funding acquisition.

Hongwei Ll: Investigation, Validation, Methodology, Review \& Editing.

Xuesong FU: Investigation, Validation, Methodology, Supervision.

Wenlong ZHOU: Resources, Project administration, Supervision.

\section{Acknowledgements}

This work was financial supported by the National Natural Science Foundation of China [grant numbers: U1908229, 52075073], and also supported by the Fundamental Research Funds for the Central Universities [grant number: DUT20GF115]. 


\section{Supplementary Material}

Supplementary material associated with this article can be found in the online version at https:// doi.org/

\section{References}

1. Yeh JW, Chen SK, Lin SJ et al (2004) Nanostructured High-Entropy Alloys with Multiple Principal Elements: Novel Alloy Design Concepts and Outcomes. Adv Eng Mater 6:299-303

2. Rost CM, Sachet E, Borman T et al (2015) Entropy-stabilized oxides. Nat Commun 6:8485

3. Miracle DB (2017) O. N. Senkov. A critical review of high entropy alloys and related concepts. Acta Mater 122:448-511

4. Sarkar A, Djenadic R, Usharani NJ et al (2017) Nanocrystalline multicomponent entropy stabilised transition metal oxides. J Eur Ceram Soc 37:747-754

5. Dragoe N, Berardan D (2019) Order emerging from disorder. Science 366:573-574

6. Bérardan D, Franger S, Dragoe D et al (2016) Colossal dielectric constant in high entropy oxides. Phys Status Solidi RRL 10:328-333

7. Wang Q, Sarkar A, Wang D et al (2019) Multi-anionic and -cationic compounds: new high entropy materials for advanced Li-ion batteries. Energy Environ Sci 12:2433-2442

8. Jimenez-Segura MP, Takayama T, Bérardan D, et al. Long-range magnetic ordering in rocksalt-type high-entropy oxides. Appl Phys Lett 2019, 114: 122401.1-122401.5

9. Chen H, Fu J, Zhang P et al (2018) Entropy-stabilized metal oxide solid solutions as CO oxidation catalysts with high-temperature stability. J Mater Chem A 6:11129-11133

10. Cardoso ALF, Perdomo CPF, Kiminami RHGA et al (2021) Enhancing the stabilization of nanostructured rocksalt-like high entropy oxide by Gd addition. Mater Lett 285:129175

11. Chen H, Jie K, Jafta CJ et al (2020) An ultrastable heterostructured oxide catalyst based on highentropy materials: A new strategy toward catalyst stabilization via synergistic interfacial interaction. Appl Catal B-Environ 276:119155

12. Mao A, Xiang H-Z, Zhang Z-G et al (2019) Solution combustion synthesis and magnetic property of rock-salt (Co0.2Cu0.2Mg0.2Ni0.2Zn0.2)O high-entropy oxide nanocrystalline powder. J Magn Magn Mater 484:245-252

13. Qin $\mathrm{M}, \mathrm{Lu} \mathrm{H}, \mathrm{Wu} \mathrm{H}$ et al (2019) Powder injection molding of complex-shaped aluminium nitride ceramic with high thermal conductivity. J Eur Ceram Soc 39:952-956

14. Wu D, Qin S, Liu H et al (2021) Enhancing piezoelectricity of PLZT ceramics by bismuth stearate coating via water-soluble defatted powder injection molding. Ceram Int 47:17956-17963

15. Fuertes V, Berges C, Gallego A et al (2020) Tailoring dielectric properties of cordierite-mullite ceramics through Ceramic Injection Moulding. Mater Sci Eng B 262:114783

16. Liu W, Wen J, Xie Z et al (2018) Powder modification mechanism, effects of binder compositions on the thermal behavior, and the mechanical properties of the ceramic injection molded system. Ceram 
Int 44:5646-5651

17. He J, Shao Z, Khan DF et al (2018) Investigation of inhomogeneity in powder injection molding of nano zirconia. Powder Technol 328:207-214

18. Zhou PP, Wu GQ, Tao Y et al (2018) Optimization of the injection molding process for development of high performance calcium oxide - based ceramic cores. Mater Res Express 5:1-10

19. Wen J, Liu W, Xie Z et al (2018) Effects of the binder compositions on the homogeneity of ceramic injection molded compacts. Ceram Int 44:3218-3225

20. Hu F, Liu W, Xie Z (2016) Surface modification of alumina powder particles through stearic acid for the fabrication of translucent alumina ceramics by injection molding. Ceram Int 42:16274-16280

21. Hnatkova E, Hausnerova B, Filip P (2019) Evaluation of powder loading and flow properties of Al203 ceramic injection molding feedstocks treated with stearic acid. Ceram Int 45:20084-20090

22. Zhao M, Qiao L, Zheng J et al (2019) Investigation of the solvent debinding in the injection molding of ZrO2 ceramics using LDEP, HDPE and wax binders. Ceram Int 45:3894-3901

23. Bianchi MF, Gameros AA, Axinte DA et al (2019) On the effect of mould temperature on the orientation and packing of particles in ceramic injection moulding. J Eur Ceram Soc 39:3194-3207

24. Izadbakhsh $\mathrm{H}$, Sheikh $\mathrm{H}$, Sharifi EM et al (2021) Low-pressure injection molding of magnesium aluminate nanoparticles: Rheological behavior and flexural properties of sintered component. Ceram Int 47:25932-25941

25. Izadbakhsh H, Sheikh H, Mohammad Sharifi E et al Development of mechanical and visible transparency properties of spark plasma sintered spinel fabricated by powder injection molding of MgAl204 nanoparticles. Ceram Int 2021

26. As A, Js B, Dn B et al (2019) Evaluation of the Technical-Economic Potential of Thermosetting Injection Moulding for Shaping of C/C-SiC-Ceramics - ScienceDirect. Procedia CIRP 85:66-71

27. Wang C, Li Z, Tian W et al Micro powder injection molding of boron carbide components with SiCAl203-Y2O3 sintering additives. Chin J Aeronaut 2020

28. Yang Xf, Yang Jh, Xu Xw et al (2015) Injection molding of ultra-fine Si3N4 powder for gas-pressure sintering. Int J Miner Metall Mater 22:654-659

29. Wiesner VL, Rueschhoff LM, Diaz-Cano Al et al (2016) Producing dense zirconium diboride components by room-temperature injection molding of aqueous ceramic suspensions. Ceram Int 42:2750-2760

30. Gromada M, Swieca A, Kostecki M et al (2015) Ceramic cores for turbine blades via injection moulding. J Mater Process Technol 220:107-112

31. Park JM, Han JS, Gal CW et al (2018) Fabrication of micro-sized piezoelectric structure using powder injection molding with separated mold system. Ceram Int 44:12709-12716

32. Wen J, Liu W, Xie Z et al (2018) Effects of PVP incorporation on the properties of injection-molded high-performance ceramics with PEG-based binders. Ceram Int 44:2718-2726 
33. Basir A, Sulong AB, Jamadon NH et al (2021) Feedstock properties and debinding mechanism of yttria-stabilized zirconia/ stainless steel 17-4PH micro-components fabricated via two-component micro-powder injection molding process. Ceram Int 47:20476-20485

\section{Figures}

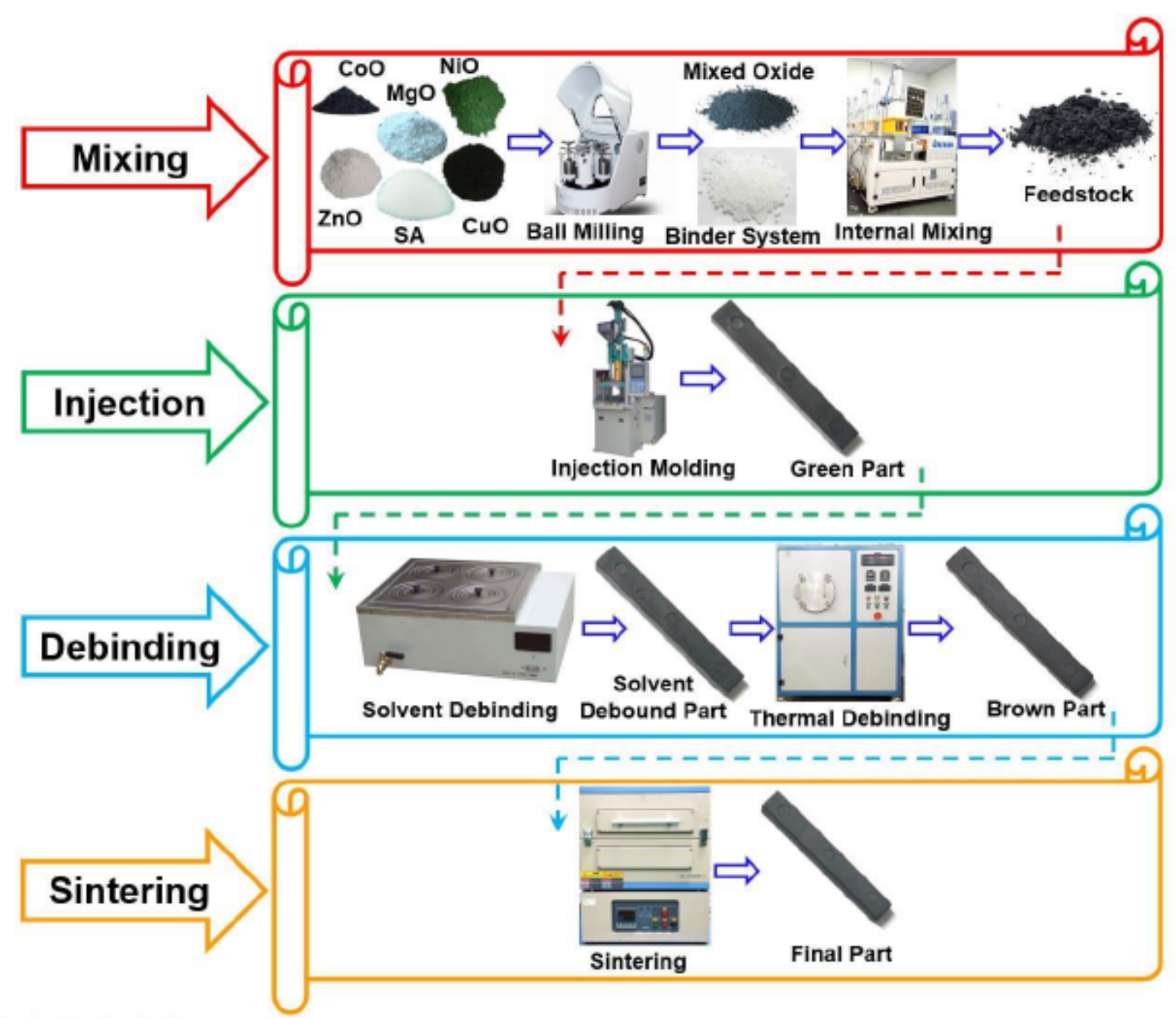

Figure 1

Schematic for preparation of (MgCoNiZnCu)O by ceramic injection molding: mixing oxide powders with organic binders, injection molding of feedstock, solvent and thermal debinding of green part, sintering of brown part. 

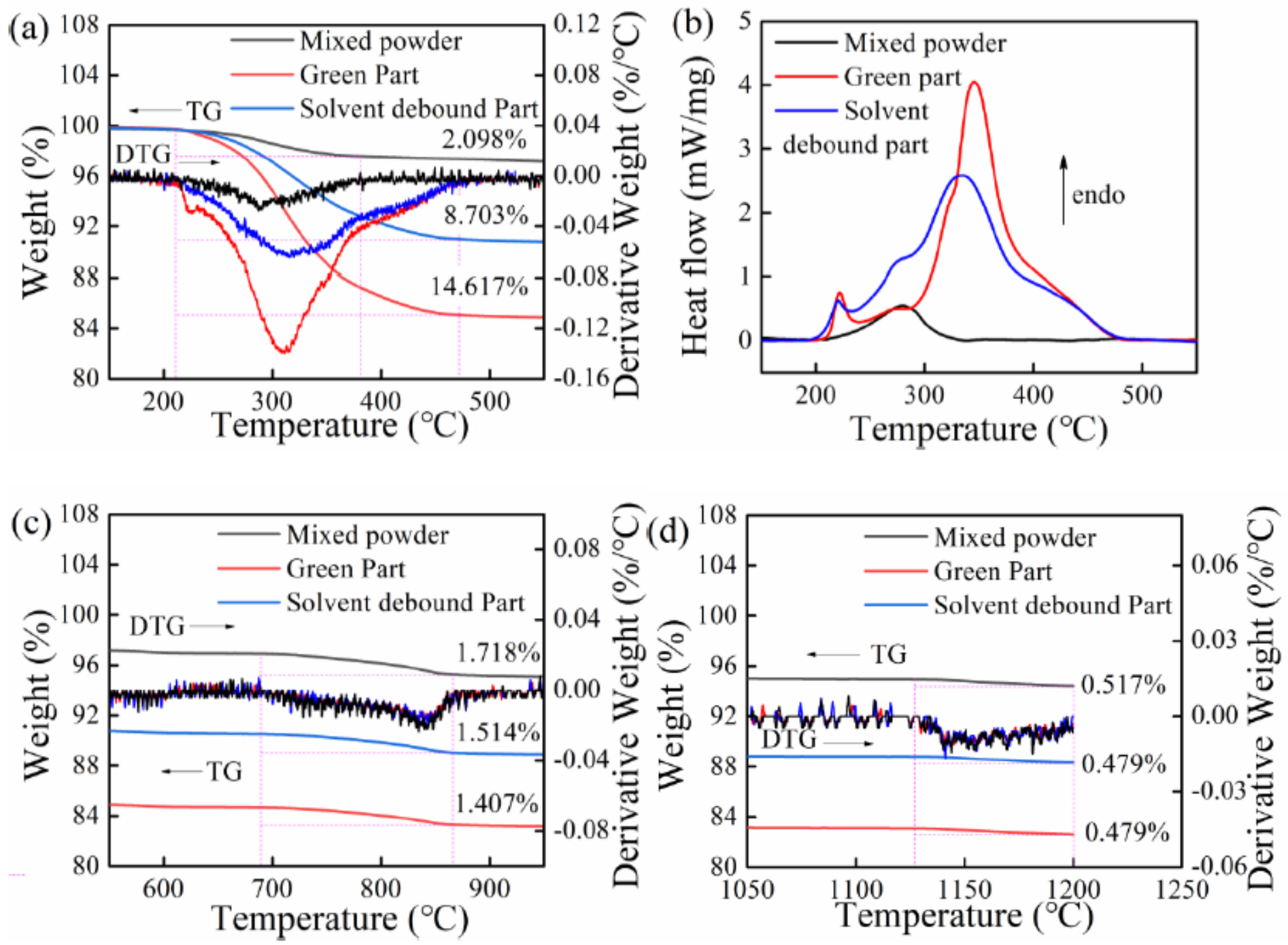

Figure 2

Thermal analysis results of mixed powder, green part and solvent debound part: (a) TG and DTG curves at $200-500{ }^{\circ} \mathrm{C}$, (b) DSC curves at $200-500{ }^{\circ} \mathrm{C}$, (c) TG and DTG curves at 600-900 ${ }^{\circ} \mathrm{C}$, (d) TG and DTG curves at $1050-1200^{\circ} \mathrm{C}$. 

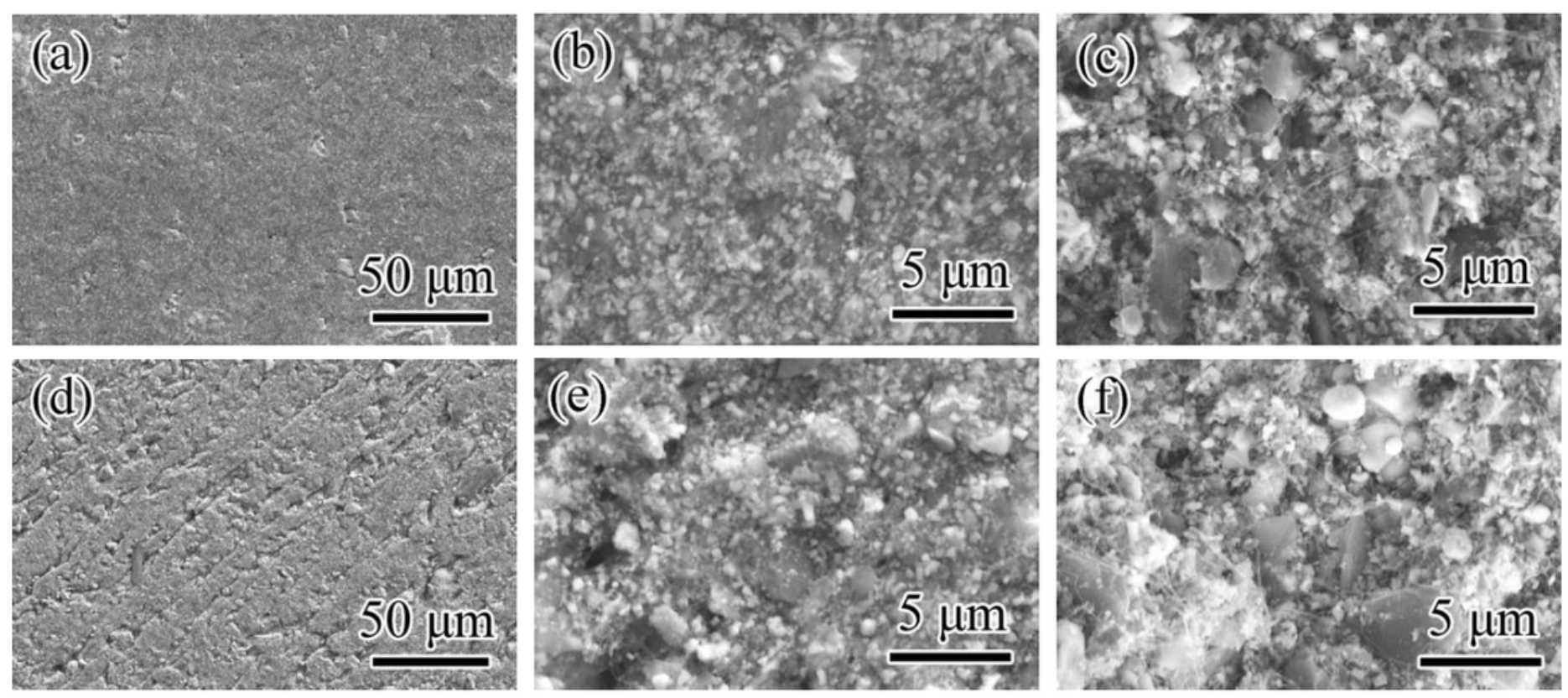

\section{Figure 3}

Morphology of SEM images: (a) surface of green part, (b) surface of green part with a higher magnitude, (c) fracture of green part, (d) surface of solvent debound part, (e) surface of solvent debound part with a higher magnitude, (f) fracture of solvent debound part. 


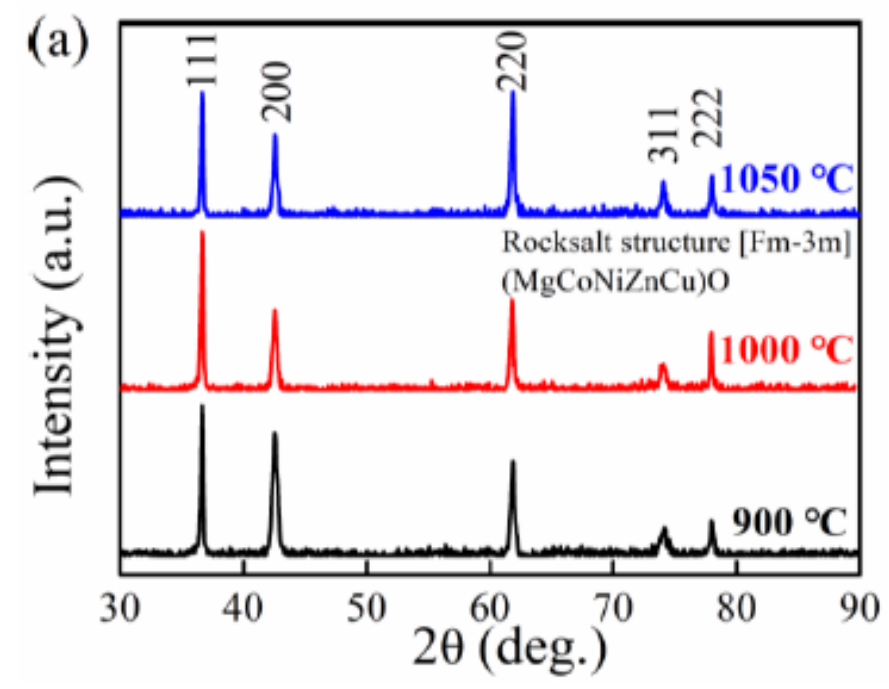

(b) $1050^{\circ} \mathrm{C}$
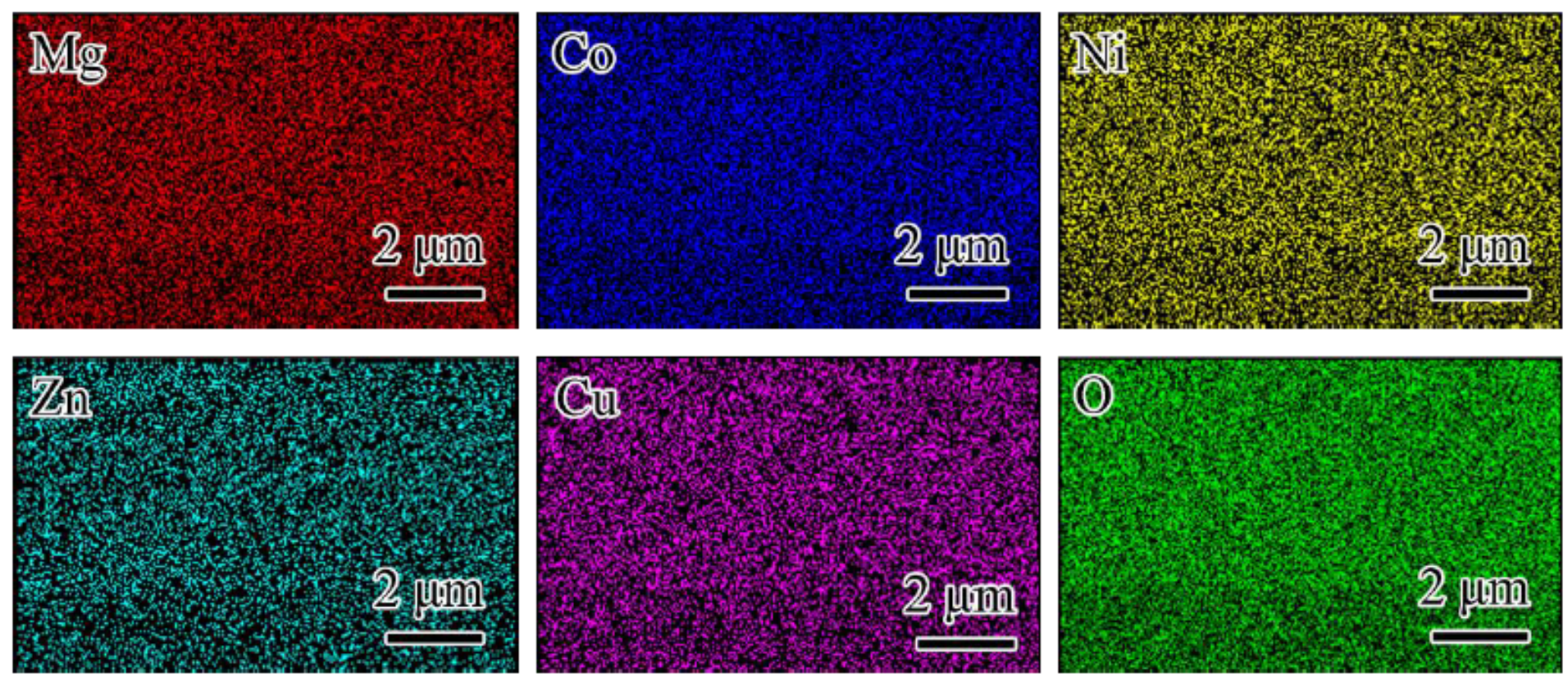

Figure 4

(a) XRD spectra of compacts sintered at 900,1000 and $1050{ }^{\circ} \mathrm{C}$, (b) SEM images of polished morphology, with the corresponding EDS mapping of $\mathrm{Mg}, \mathrm{Co}, \mathrm{Ni}, \mathrm{Zn}, \mathrm{Cu}$ and $\mathrm{O}$ elements synthesized at $1050{ }^{\circ} \mathrm{C}$. 

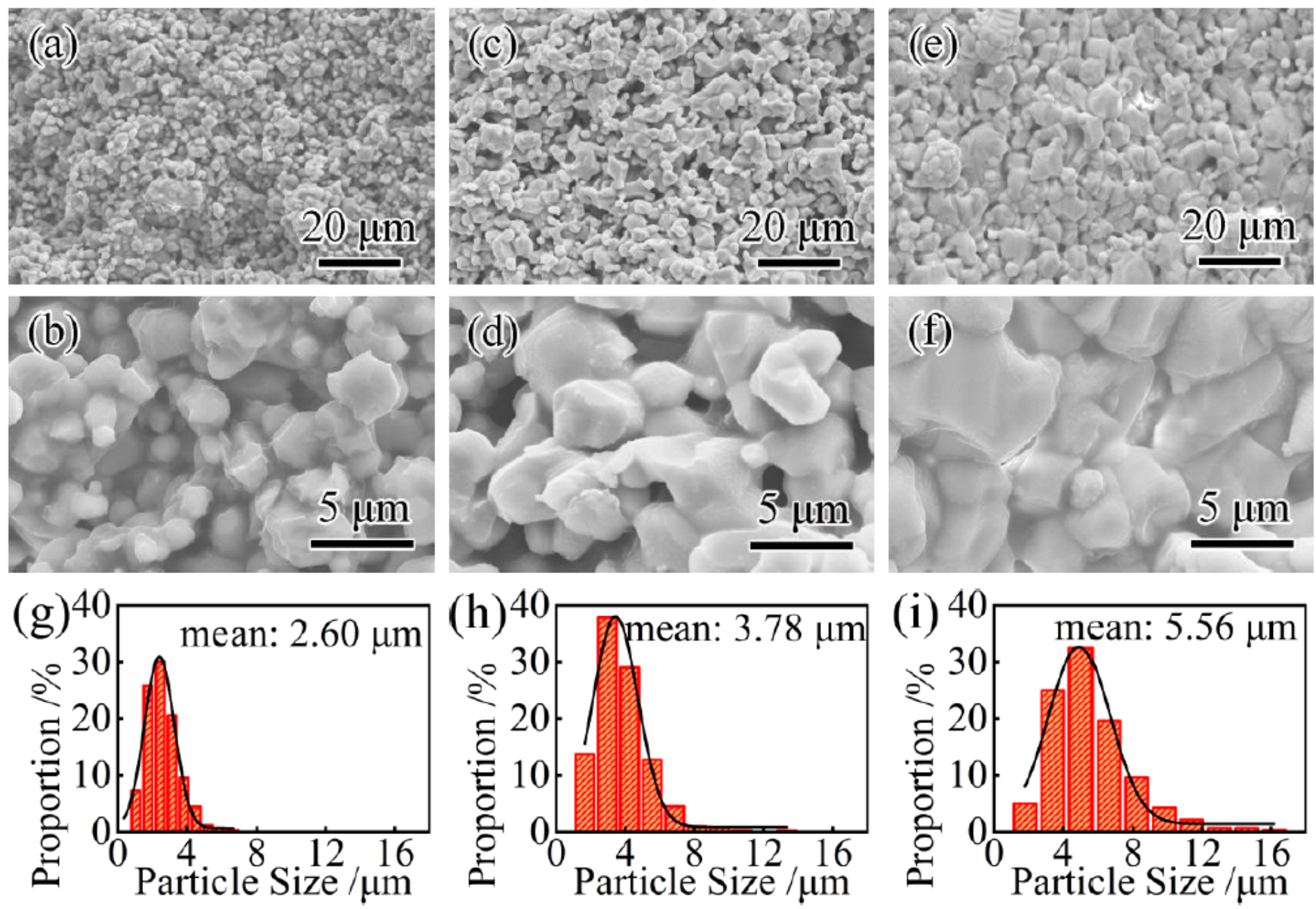

Figure 5

Fractured micro-graph of CIM sintered compacts: $(a, b)$ morphology synthesized at $900{ }^{\circ} \mathrm{C},(\mathrm{c}, \mathrm{d})$ morphology synthesized at $1000{ }^{\circ} \mathrm{C},(\mathrm{e}, \mathrm{f})$ morphology synthesized at $1050{ }^{\circ} \mathrm{C}$. Grain size distribution at different sintering temperature: (g) $900{ }^{\circ} \mathrm{C}$, (h) $1000{ }^{\circ} \mathrm{C}$, (i) $1050{ }^{\circ} \mathrm{C}$. It should be noted that grain size of pellets (g), (h), (i) was estimated using nano measure software from (a), (c), (e), respectively.

\section{Supplementary Files}

This is a list of supplementary files associated with this preprint. Click to download.

- SupplementaryMaterial.doc 